\title{
Evaluación de los estudios de Documentación: el caso de la Universidad Politécnica de Valencia
}

\author{
Por Álvaro Doménech, Alejandro Juan-Argomániz, José Llorens, José-Antonio Ontalba-Ruipérez, \\ Antonio Pérez-Gómez, Andrés Terrasa y Eduardo Vendrell
}

\begin{abstract}
Resumen: Se describe el proceso de evaluación de la Licenciatura de Documentación de la Universidad Politécnica de Valencia (UPV). Se explica el desarrollo de los estudios académicos de Información y Documentación en España, el procedimiento de evaluación de enseñanzas universitarias según la agencia española responsable (Aneca) y, finalmente, se exponen los resultados más destacados de dicha evaluación de los estudios de Documentación de la UPV.
\end{abstract}

Palabras clave: Estudios universitarios, Licenciatura, Evaluación, Calidad.

Title: Evaluation of the Library and Information Science degree of the Polytechnical University of Valencia

Abstract: The evaluation process of the Library and Information Science's (LIS) Degree of the Polytechnic University of Valencia is described. The development of the Spanish LIS's academic studies and the university education evaluation procedure according to the responsible Spanish agency (Aneca) are explained; and, finally, the most noticeable results of the evaluation of the UPV Documentation's studies are exposed.

Keywords: University studies, Academic degree, Evaluation, Quality.

Doménech, Álvaro; Juan-Argomániz, Alejandro; Llorens, José; Ontalba-Ruipérez, José-Antonio; Pérez-Gómez, Antonio; Terrasa, Andrés; Vendrell, Eduardo. "Evaluación de los estudios de Documentación: el caso de la Universidad Politécnica de Valencia”. En: El profesional de la información, 2007, septiembre-octubre, v. 16, n. 5, pp. 475-480.

DOI: $10.3145 /$ epi.2007.sep.10

EN NO POCAS OCASIONES se plantea en foros $y$ congresos la adecuación y validez de los estudios universitarios de Documentación a la realidad profesional y laboral de archiveros, bibliotecarios, documentalistas y demás profesionales de la información. En ésa y otras líneas, la Agencia Nacional de Evaluación de la Calidad y Acreditación (Aneca) lleva a cabo el Programa de Evaluación Institucional (PEI). A lo largo del año 2006 y hasta principios de 2007 se ha llevado a cabo en la Facultad de Informática de la Universidad Politécnica de Valencia (UPV) la evaluación de la Licenciatura en Documentación.

\section{Estudios universitarios de documentación}

Con el precedente de la $E s$ cuela Superior de Bibliotecarias en Barcelona, creada por la Mancomunidad de Cataluña en 1915, el real decreto 3104/1978, de 1 de diciembre, crea las enseñanzas de biblioteconomía y documentación dentro del sistema universitario español. Más adelante, la orden de 24 de febrero de 1981 establece las directrices para la elaboración de los planes de estudio de las escuelas universitarias de biblioteconomía y documentación como titulación de primer ciclo. La de Barcelona fue la primera, empezando en el curso 1982-1983. Con todo ello se quería dar salida a las necesidades de formación académica que, además, permitiera el acceso a unos perfiles profesionales identificados en las relaciones de puestos de trabajo.

Diez años después del primer plan de estudios oficial de la diplomatura, el real decreto 912/1992, de 17 de julio, establece el título universitario oficial de Licenciado en Documentación, como titulación de segundo ciclo, y las directrices generales propias de los planes de estudios conducentes a su obtención. Las primeras universidades que incluyeron la licenciatura en documentación en su oferta docente fueron Alcalá de Henares, Carlos III de Madrid, Granada y Salamanca, empezando todas ellas en el curso 1994-1995. 
En el año escolar 1996-1997, la Universidad de Valencia ofrece la Diplomatura en biblioteconomía y documentación (publicándose su plan de estudios en la resolución de 31 de octubre de 1996, BOE (Boletín Oficial del Estado) de fecha 15 de febrero de 1997). Un año más tarde, en el curso 1997-1998, la $U P V$ incluye por primera vez la Licenciatura en documentación en su oferta docente (resolución de 19 de diciembre de 1996, BOE de 16 de enero de 1997). La $U P V$, a la hora de diseñar y orientar la titulación, considera que estos estudios están próximos a los de informática y, por tanto, la Facultad de Informática acaba siendo el centro que los acoge. De hecho, los estudios de licenciado en documentación en la $U P V$ están dotados de un fuerte carácter informático como rasgo característico diferenciador.

En esa línea podrían aducirse algunos argumentos, de los que destacan:

- El objeto de estudio: ya en el siglo XXI es indudable la preeminencia del documento electrónico. No puede obviarse la importancia de los documentos en papel, pero, cada vez más, habrá que considerar casi siempre que el original es el electrónico y el que se maneja en papel es sólo una copia.

- Las técnicas aplicadas en la producción, organización, intercambio y gestión de documentos son, en la mayoría de ocasiones, las de la informática.

- La evolución, la asimilación de nuevos métodos, estándares, procedimientos... que van apareciendo, apadrinados por organizaciones mundiales, y que suelen tener un sustrato tecnológico que hay que tener asimilado previamente para comprender el alcance de las nuevas propuestas.

En cuanto a la población de estudiantes a los que se ha enfocado esta titulación desde el principio, además de los diplomados en biblioteconomía y documentación y licenciados en varias carreras (básicamente, humanísticas y sociales), se da también el rasgo idiosincrático de los ingenieros técnicos en informática de gestión. Para ellos, la Licenciatura en documentación es una de las posibles continuaciones naturales de su formación académica y profesional.

\section{La evaluación de las enseñanzas universitarias}

En lo que a las universidades españolas se refiere, en la década de los 90 hubo cuatro acciones referidas a evaluación institucional previas a la actual:

- Programa experimental de evaluación de la calidad del sistema universitario (1992-94). Promovido por el Consejo de Universidades e implicó a 17 universidades.

- Proyecto piloto europeo para la evaluación de la calidad de la enseñanza superior (1994-95). Se realizó a escala europea e implicó a 44 universidades.

- Plan nacional de evaluación de la calidad de las universidades (Pnecu) 1995-2000. Fue el impulsor de la evaluación de la calidad de las universidades a nivel institucional. Elaboró una metodología común y facilitó los instrumentos necesarios para la recogida de datos y las guías para llevar a cabo el proceso de evaluación basado en el modelo de excelencia europea EFQM (European Foundation for Quality Management).

\section{http://www.efqm.org/}

- II Plan de la calidad de las universidades (2000-2002). Siguió la línea anterior.

Con la Ley orgánica de universidades (LOU, diciembre 2001), que dedica su título $\mathrm{V}$ a la Evaluación de las universidades, se crea la Aneca (Agencia Nacional de Evaluación de la Calidad y Acreditación).

\section{"Con la LOU (diciembre \\ 2001) se crea la Aneca, que gestiona desde entonces los programas de evaluación de las titulaciones universitarias"}

\section{http://www.aneca.es}

Desde entonces los programas de evaluación de las titulaciones universitarias son gestionados por esta agencia.

En este contexto, el PEI (Programa de evaluación institucional) tiene como objetivo facilitar un proceso de evaluación para la mejora de la calidad de las enseñanzas conducentes a la obtención de un título universitario de carácter oficial. Requiere dos comités, el Comité de autoevaluación (CA) y el Comité de evaluación externa (CEE), e implica tres niveles institucionales:

- La Aneca centraliza la gestión del proceso, nombra el $C E E$, suministra las directrices básicas de procedimiento y publica los resultados.

- La universidad donde se cursa el título ha de disponer de un servicio propio de planificación y gestión de calidad que proporcione la Guía de evaluación y realice funciones de asesoría metodológica a los interesados.

- El centro donde se imparte la titulación ha de nombrar el $C A$ y suministrar la infraestructura necesaria para el trabajo.

\footnotetext{
"El PEI facilita el proceso de evaluación para optimizar la calidad de las enseñanzas para la obtención de un título universitario oficial"
} 
El PEI está organizado en tres fases. Cada una es responsabilidad de un Comité que se hace cargo de elaborar un informe. Éstos constan de dos partes: el primero trata de hacer un análisis objetivo y riguroso de la situación, y el segundo incluye las propuestas de mejora, identificación de prioridades, procedimientos y niveles institucionales implicados. Cada parte del informe cubre seis áreas (Programa formativo, Organización de la enseñanza, Recursos humanos, Recursos materiales, Proceso formativo, y Resultados) que definen los aspectos más relevantes a valorar durante la evaluación. A continuación se describen las tres fases de un PEI:

\section{Fase 1: Autoevaluación}

Es una etapa que ofrece a la comunidad universitaria la oportunidad de reflexionar y valorar el estado de funcionamiento de una titulación. Cumple además una función práctica, ayudando a detectar los puntos fuertes y débiles, con vistas a la creación de planes y estrategias de mejora.

Es realizada por el $C A$, compuesto por un grupo de representantes de las diferentes categorías profesionales de la titulación y capaz de movilizar a toda la comunidad para que participe en el PEI. Sus tareas son analizar y valorar todas las áreas indicadas en la Guía describiendo y evaluando su situación inicial respecto a los criterios establecidos, coordinar la recogida de información necesaria, alcanzar consensos sobre el valor de los resultados obtenidos y proponer posibles acciones de mejora.

El resultado es el Informe de autoevaluación (IA), en el que se valoran los subcriterios de cada una de las seis áreas a partir de una serie de evidencias documentales que hay que reunir físicamente, se resumen fortalezas y debilidades, y se incluyen sugerencias de mejora. La herramienta más utilizada a estos efectos es el modelo EFQM.
El IA se divulga por el resto de la comunidad para recabar posibles alegaciones.

\section{Fase 2. Evaluación externa}

Consiste en un diagnóstico para determinar si las actividades y resultados de la titulación evaluada cumplen los requisitos de calidad previamente establecidos, y si éstos son implantados de forma efectiva y son adecuados para alcanzar los objetivos fijados. El objetivo fundamental de esta fase es la validación del $I A$, a través del análisis documental y de una visita al centro, para constatar cómo se sitúa la titulación evaluada respecto al modelo de evaluación.

De ello se encarga el $C E E$, que es un grupo de evaluadores externos compuesto por personas de reconocido prestigio y competencia en las áreas bajo la responsabilidad de la titulación evaluada.

El resultado es el Informe de evaluación externa, que remite la Aneca a la Universidad para difundirlo entre el personal y de los grupos interesados en la evaluación.

Fase 3. Informe final y plan de mejoras

A partir del Informe de evaluación externa, el $C A$ elabora un borrador del Informe final de autoevaluación y lo expone públicamente antes de elaborar la versión definitiva. Se trata de un documento informativo sobre los resultados del proceso de evaluación, tomando como base el $I A$ y las sugerencias incluidas en el Informe de evaluación externa.

Ahora bien, la evaluación no finaliza aquí, sino que continúa con un proceso de seguimiento que garantice que los cambios propuestos se llevan a la práctica con éxito. Así, el informe final debe contener un Plan de mejoras (siguiendo con la metodología de la Aneca para ello) que especifique cómo se comprobará periódicamente la ejecución de las acciones implantadas, qué procedimientos se seguirán para diseñar nuevas acciones de perfeccionamiento y cómo se garantizará que el sistema de calidad continúe activo. En este sentido, el PEI conlleva como uno de sus objetivos asociados la instauración de una cultura de evaluación continua para la optimización de la titulación impartida en un centro universitario.

\section{Proceso de evaluación de la Licenciatura en Documentación de la UPV}

El Proceso de autoevaluación comenzó por la selección del $C A$ por la dirección del centro a partir de unos criterios planteados por la Guía y bajo la supervisión de la Agencia de Calidad de la UPV. Una vez decidida la composición, fue aprobada en la Comisión Permanente (delegada de la Junta de Centro) de la Facultad de Informática el 23 de diciembre de 2005. Así, los autores del presente trabajo se configuraron en $C A$ para la titulación de Licenciatura de Documentación de la $U P V$.

Tras una fase de formación inicial a cargo de la Agencia de Calidad de la UPV, el Comité se constituyó formalmente y estableció un calendario de reuniones (de mediados de enero a principios de mayo de 2006) y una metodología de trabajo.

En la planificación de las reuniones también se incluyó, de forma aproximada, qué subcriterios de la Guía de autoevaluación debían evaluarse en cada reunión para poder cumplir con el calendario previsto.

La metodología de trabajo de las reuniones fue la siguiente: para cada subcriterio a evaluar se consideraron las evidencias con las que se contaba llegando a un acuerdo respecto a la contextualización de éste, a la valoración de su cumplimiento y al plan de mejora propues- 
to, en su caso. Tras la discusión de todos los subcriterios, el $C A$ se reservó varias reuniones para la revisión del borrador final del $I A$.

Finalizada la fase de elaboración del informe, el texto estuvo público del 16 al 25 de mayo de 2006 para que las personas relacionadas con la titulación pudieran hacer indicaciones. Cumplido el plazo, el $C A$ se reunió para incluir los comentarios recibidos y ultimar el $I A$, que fue enviado a la Aneca en el plazo previsto, el 31 de mayo de 2006 .

\section{Evaluación externa}

La segunda fase del proceso supuso la visita de un $C E E$ los días 26 y 27 de octubre de 2006, siendo las personas elegidas María Pinto Molina (catedrática de documentación de la Universidad de Granada) y José Antonio Moreiro González (catedrático de documentación de la Universidad Carlos III de Madrid).

El CEE se reunió por separado con una representación de cada uno de los colectivos relacionados con la titulación: $C A$, dirección del centro, directores de los departamentos vinculados a la titulación, profesorado, alumnado, personal de administración y servicios, y egresados. Además, convocó una audiencia abierta a cualquier persona relacionada con la titulación, y visitó las instalaciones del centro.

Un mes después el $C E E$ envío su informe, a través de la Aneca, al presidente del $C A$ y se abrió un

\section{"EI PEI ha revelado cierta falta de revisión de los contenidos e incluso de la estructura del plan de estudios, cosa que es habitual, por otra parte, en el conjunto de las carreras universitarias"}

plazo para enviar alegaciones a dicho informe. Se difundió el informe entre los implicados en el proceso y se recabaron sus opiniones. Finalmente, fue considerado correcto (la mayoría de sus conclusiones coincidían con las del IA respecto a los principales puntos fuertes y débiles de la titulación) y no se produjo ningún comentario ni alegación al mismo.

\section{Plan de mejoras}

El Plan de mejoras de la titulación se redactó durante los meses de enero y febrero de 2007 (el plazo para su envío a la Aneca era el 28 de febrero).

Se debatieron y evaluaron los criterios, y el resultado del trabajo se puso en común, revisando cada una de las mejoras propuestas y elaborando un documento provisional con el Plan. Los comentarios del $C A$ al borrador fueron recogidos en la versión definitiva del Plan de mejoras.

Independientemente de la metodología formal establecida en el protocolo de la Aneca, el $C A$ se planteó la selección y diseño de las acciones de mejora desde tres principios estratégicos básicos:

-Incidir principalmente en acciones relativas a las debilidades detectadas en la titulación por el $C A$ y/o por el $C E E$ y, en especial, en aquellas que resulten realmente abordables.

-Mantener la responsabilidad de las acciones dentro de la titulación y del centro donde se imparte (la Facultad de Informática).

-Plantear los cambios en dos plazos: el primero entre el final del proceso (febrero de 2007) y el principio del curso 2007-2008, y el segundo durante el año escolar 2007-2008. Se desestimó acometer acciones a más largo plazo por la situación actual de las titulaciones españolas, pendientes de ser sustituidas por las nuevas creadas en el marco del Espacio Europeo de Educación Superior antes de 2010.

\section{Resultados}

Aunque lo que se busca con todo este procedimiento es, principalmente, identificar los aspectos perfeccionables de la titulación para rentabilizar así la gestión de los estudios, deben mencionarse también los puntos fuertes resaltados por el $P E I$ sin caer por ello en la autocomplacencia. Así, entre las fortalezas, destaca el elemento diferenciador basado en el componente tecnológico de la titulación y, relacionado con ello, la adecuada preparación de los egresados para las exigencias laborales, especialmente en el dominio de habilidades tecnológicas.

A continuación, se comentan los aspectos que requieren de mejoras siguiendo la estructura de áreas de la Guía de evaluación seguida a lo largo de todo el proceso:

\section{Programa formativo}

Se ha advertido un déficit de optatividad en el plan de estudios (un $26,6 \%$ ), con lo que se lo debería dotar de intensificaciones curriculares coherentes con los distintos perfiles de egreso.

El PEI ha revelado, asimismo, una cierta falta de revisión de los contenidos e incluso de la estructura del plan de estudios. Esta carencia es habitual en las carreras universitarias, por más que la adecuación de la oferta académica a la demanda social y laboral sea obvia y la revisión periódica del plan de estudios se considere un procedimiento necesario.

\section{Organización de la enseñanza}

En esta área se ha destacado la ausencia total de participación e implicación del alumnado de la Licenciatura de Documentación de la $U P V$ en los foros habituales de participación de la Facultad (como las diferentes comisiones o la $\mathrm{De}$ - 
legación de alumnos). Ello provoca que desde la dirección del centro no se tenga conciencia de sus opiniones y su grado de satisfacción con los procesos del programa formativo, y que se pueda caer en un bucle repetible en los futuros grados $\mathrm{y}$ postgrados.

\section{"Se ha observado la ausencia total de participación e implicación del alumnado de la Licenciatura de Documentación de la UPV en los foros habituales de la Facultad"}

\section{Recursos humanos}

Falta profesorado específico de Documentación y, sobre todo, existe un desequilibrio en su plantilla que lleva a que los profesores asociados a tiempo parcial impartan más del $60 \%$ de los créditos.

\section{Recursos materiales}

Escasez de espacios de trabajo común: una única aula para toda la Facultad (es decir, para las Licenciaturas en Informática y en Documentación).

Ha aflorado que la biblioteca no dispone de suficientes recursos de información (hemeroteca, mediateca, colección bibliográfica, bases de datos, etc.), pero que, en parte, responde a la falta de desideratas de los profesores; $y$, sobre todo, la escasa consulta y préstamo por parte de los alumnos. En este aspecto hay que considerar que rara vez la docencia requiere del alumnado la consulta de artículos, monografías $o$, especialmente, bases de datos; esto es debido, seguramente, a que los materiales suministrados por el profesorado son lo suficientemente completos como para que los alumnos obvien, por lo general, la consulta directa de las fuentes.

\section{Proceso formativo}

No hay ni un proceso sistematizado de captación de alumnos en los contextos adecuados (principalmente en la Diplomatura de Biblioteconomía en la Universidad de Valencia, $U V$ ) ni acciones de acogida para los alumnos de nuevo ingreso (la $U P V$ sólo las contempla en titulaciones de primer ciclo)

Además, se percibe una reticencia por parte de algunos profesores a incorporar nuevas metodologías docentes en sus asignaturas. Al margen de otras causas (generales o particulares), es manifiesta la preeminencia cada vez mayor que la Universidad española está dando a la investigación frente a la gestión y la docencia, con las consecuencias negativas que ello conlleva (principalmente para esta última).

\section{Resultados}

Se ha comprobado que los resultados del programa formativo no se tienen en cuenta de manera adecuada para establecer acciones de mejora y revisión del mismo, con lo que se cae en el riesgo de que los estudios no se estén adaptando ni ajustando a la demanda de la sociedad (otra cosa es la visibilidad profesional que tenga el titulado en documentación o que esa sociedad sea consciente de algunas de sus necesidades, como se ha dicho antes).

De forma importante, se constata la poca visibilidad de la titulación tanto dentro de la $U P V$ como fuera de ella, y, en especial, la falta de coordinación con los estudios de la Diplomatura de la $U V$.
A lo expuesto hasta ahora habría que añadir la caída del número de alumnos (de 73 matriculados de nuevo ingreso en 2003-2004 a 63 en 2004-2005), que debe ser contextualizada por medio de una serie de factores externos: por un lado, un descenso en la natalidad de la segunda mitad de los años 80 que afecta a toda la Universidad española; por otro, cierta saturación de la demanda laboral de profesionales de la información requerida, básicamente, por la administración pública (no en el caso de la Comunidad Valenciana, donde el sistema público de archivos, bibliotecas y centros de documentación requiere aún de desarrollo y consolidación); $\mathrm{y}$, finalmente, por la lentitud en el cambio del modelo económico español y la falta de conciencia de la empresa de la necesidad de que los profesionales de la información formen parte de su organigrama. En cualquier caso, el alumno universitario empieza a ser considerado, cada vez más, como cliente.

Todas las carencias no son, en la mayoría de casos, debilidades propias de la $U P V$, sino que son comunes (a veces como característica endémica) a la Universidad española. Sería de interés que se publicaran los resultados de otros $P E I$ s con el fin de identificar desde este enfoque perfiles según departamentos, titulaciones, universidades o áreas geográficas.

\section{Conclusiones}

Como ya se ha reseñado, un Programa de evaluación institucional tiene como objetivo fundamental
"El número de alumnos disminuye debido al descenso en la natalidad de la segunda mitad de los años 80 , a cierta saturación de la demanda laboral de profesionales de la información, y a la lentitud en el cambio del modelo económico español" 
establecer unas pautas de revisión y mejora de la calidad de las enseñanzas conducentes a la obtención de un título universitario. Partiendo de ese objetivo, el de la titulación de Licenciado en documentación impartida en la Facultad de Informática de la $U P V$ ha permitido obtener unos puntos fuertes en la organización de las enseñanzas, así como unos puntos en los que hay que actuar.

La Facultad de Informática ha establecido un calendario para su ejecución, priorizando en el tiempo las de mayor importancia para la organización de las enseñanzas.

El PEI detallado en el presente trabajo ha derivado en una toma de conciencia del estado y la perspectiva futura de la Licenciatura en documentación en la $U P V$. Este hecho es importante en un momento en el que se considera la implantación de los nuevos títulos de grado y postgrado a nivel estatal.
Como punto final, gracias a la participación en un PEI la comunidad de la titulación toma conciencia de un proceso de mejora continua de la calidad de las enseñanzas. Es de esperar que ello aumente el vínculo de alumnos, profesores y personal de administración y servicios con la titulación y el centro en el que está implantada, factor básico en el resultado final de la impartición de las enseñanzas.

\section{Bibliografía}

Abadal, Ernest. "Espacio europeo de formación superior en Biblioteconomía y Documentación". En: Anuario ThinkEPI 2007, 2006, pp. 123-126.

Agencia Nacional de Evaluación de la Calidad y Acreditación. Programa de Evaluación Institucional. Guía de Autoevaluación 2005-2006. Madrid: Aneca, 2005.

http://www.aneca.es/active/docs/pei_0506_guiaautoevaluacion.pdf [Consulta: 18 julio 2007]

Ferrer-Sapena, Antonia; Lloret, Nuria; Peset, Fernanda. "El aprendizaje desde la práctica. Integración de nuevas metodologías docentes en la Licenciatura de Documentación de la Universidad Politécnica de Valencia". En: VIII Encuen- tros Internacionales sobre Sistemas de Información y Documentación (Ibersid 2003) [Consulta: 26-7-2007].

http://eprints.rclis.org/archive/00000385

Ramos-Fajardo, Carmen. "Profesionales de la Información en España: Ayer, hoy, mañana". En: Documentación de las ciencias de la información, 1993, nº 16, pp. 169-176.

Título de grado en Información y Documentación. Madrid: Aneca, 2004.

http://www.aneca.es/modal_eval/docs/conver_ biblio.pdf [Consulta: 18 julio 2007]

Álvaro Doménech, Alejandro Juan-Argomániz, José Llorens, José-Antonio Ontalba-Ruipérez, Antonio Pérez-Gómez, Andrés Terrasa y Eduardo Vendrell

Facultad de Informática, Universidad Politécnica de Valencia.

alvaro@disca.upv.es

aljuaar@fiv.upv.es

jllorens@dsic.upv.es

joonrui@upv.es

aperez@upvnet.upv.es

aterrasa@dsic.upv.es

even@isa.upv.es

\section{IraLIS}

Por iniciativa de esta revista EPI, el Directorio EXIT, el repositorio E-LIS y la Univ. Politécnica de Valencia, en febrero de 2007 se puso en marcha el International Registry for Authors in Library and Information Science con el fin de estandarizar las firmas e identificar así mejor a cada autor, en especial a los de habla hispana. IraLIS también pretende que las bases internacionales citen adecuadamente a los autores.

A partir del núm. 3, mayo-junio 2007 (vol. 16) EPI aplica el formato iralis a los nombres de todos los autores, tanto firmantes como los que aparecen citados en las bibliografías.

\section{http://www.iralis.org/}

Desde estas páginas te invitamos a que te "iralices", regitrándote en la mencionada web, aunque todavía no hayas publicado nada.

IraLIS está respaldado por un Comité Asesor entre los que se encuentran: Isidro F. Aguillo (Cindoc), José Manuel Báez-Cristóbal (Fecyt), María Bordons (Cindoc), Jordi Camí-Morell (Parc de Recerca Biomèdica de Barcelona), Emilio Delgado López-Cózar (Universidad de Granada, EC3), Isabel Gómez-Caridad (Cindoc), Vicente P. Guerrero-Bote (Universidad de Extremadura), Félix de MoyaAnegón (Universidad de Granada, SClmago) y Elías Sanz-Casado (Universidad Carlos III de Madrid). 